\title{
Elliptic Flow at SPS and RHIC: From Kinetic Transport to Hydrodynamics
}

\author{
P.F. Kolb ${ }^{a-c}$, P. Huovinen ${ }^{d}$, U. Heinz ${ }^{a-c}$, and H. Heiselberg ${ }^{e}$ \\ ${ }^{a}$ Theoretical Physics Division, CERN, CH-1211 Geneva 23, Switzerland \\ ${ }^{b}$ Institut für Theoretische Physik, Universität Regensburg, D-93040 Regensburg, Germany \\ ${ }^{c}$ Department of Physics, The Ohio State University, 174 West 18th Ave., Columbus, OH 43210, USA \\ ${ }^{d}$ Lawrence Berkeley National Laboratory, Berkeley, CA 94720, USA \\ ${ }^{e}$ NORDITA, Blegdamsvej 17, DK-2100 Copenhagen Ø, Denmark
}

(November 2, 2018)

Anisotropic transverse flow is studied in $\mathrm{Pb}+\mathrm{Pb}$ and $\mathrm{Au}+\mathrm{Au}$ collisions at SPS and RHIC energies. The centrality and transverse momentum dependence at midrapidity of the elliptic flow coefficient $v_{2}$ is calculated in the hydrodynamic and low density limits. Hydrodynamics is found to agree well with the RHIC data for semicentral collisions up to transverse momenta of $1-1.5 \mathrm{GeV} / c$, but it considerably overestimates the measured elliptic flow at SPS energies. The low density limit LDL is inconsistent with the measured magnitude of $v_{2}$ at RHIC energies and with the shape of its $p_{t}$-dependence at both RHIC and SPS energies. The success of the hydrodynamic model points to very rapid thermalization in $\mathrm{Au}+\mathrm{Au}$ collisions at RHIC and provides a serious challenge for kinetic approaches based on classical scattering of on-shell particles.

PACS numbers: 25.75-q, 24.85.+p, 25.75.Ld

Keywords: Relativistic heavy-ion collisions; elliptic flow

1. Introduction.- Anisotropic flow has been measured in relativistic nuclear collisions 11 , and calculations, in particular for collisions at SPS and RHIC energies, exist within a variety of frameworks: hydrodynamics [6-10], the low density limit of kinetic theory 11 , parton cascades [12,13], hadronic cascade codes 14 19], combinations thereof 20], and jet quenching [21]. Recent detailed SPS [3,4] and RHIC [5] data on the centrality and transverse momentum dependence of pion and proton elliptic flow begin to discriminate between different model calculations and to yield quantitative insights into initial conditions, compression, rescattering time scales, expansion dynamics and possible phase transitions during the expansion stage of the reaction zone.

Cascade calculations based on the incoherent scattering of classical on-shell particles and the low density limit of classical kinetic theory are expected to work best for peripheral collisions where the density of produced particles is sufficiently low and only a few rescatterings occur. Central collisions produce higher particle densities where the hydrodynamic limit may be more suitable. One of the most interesting questions in the kinetic theory of relativistic heavy ion collisions is where and how the transition between the dilute and dense limits happens.

In this paper we present detailed calculations of the impact parameter and transverse momentum dependence of elliptic flow in a hydrodynamic model [7,8] and in the low density limit (LDL) of Ref. [11], and we compare the results to recent SPS and RHIC data [3 5]. We test the validity of these two models in semi-central $\mathrm{Pb}+\mathrm{Pb}$ and $\mathrm{Au}+\mathrm{Au}$ collisions where the elliptic flow signal is large and thus presents a perfect tool.

2. Geometry and Anisotropic Flow.- Consider two nuclei of radius $R$ colliding at impact parameter $b$. We refer to the collision as "central" when $b \lesssim \frac{1}{2} R$, "semi-central" when $\frac{1}{2} R \lesssim b \lesssim \frac{3}{2} R$, and "peripheral" when $b \gtrsim \frac{3}{2} R$. In non-central collisions the initial overlap zone is narrower parallel than perpendicular to the impact parameter. A simple ansatz for the initial density in the interaction region is that it scales with the number of participating nucleons per unit area in the transverse plane [6 9 . This predicts a nearly linear dependence of the multiplicity on $N_{\text {part }}$ in central and semi-central $\mathrm{Au}+\mathrm{Au}$ collisions at RHIC [22]. Other initializations have been proposed 23]. The sensitivity of elliptic flow to the details of the initialization is studied elsewhere 222].

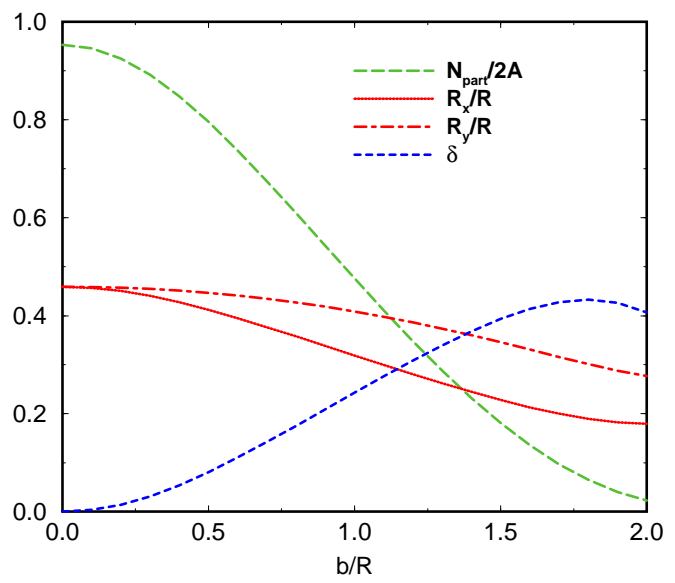

FIG. 1. The number of participants $N_{\text {part }}$, the initial transverse radii $R_{x}, R_{y}$, and the initial spatial deformation $\epsilon_{x} \equiv \delta$ for $\mathrm{Au}+\mathrm{Au}$ at $\sqrt{s}=130 \mathrm{AGeV}$, as functions of the scaled impact parameter $b / R$. For details see text.

The nuclear thickness function entering the calculation of the initial conditions [6 8] is evaluated with realistic Woods-Saxon nuclear density profiles for the incoming nuclei, with radius $R=1.12 A^{1 / 3}-0.86 A^{-1 / 3} \mathrm{fm}(A=207$ for $\mathrm{Pb}+\mathrm{Pb}$ at the SPS and $A=197$ for $\mathrm{Au}+\mathrm{Au}$ at RHIC) and standard surface diffuseness $a=0.54 \mathrm{fm}[7]$. The nucleon-nucleon cross section is taken as $32 \mathrm{mb}$ at $\sqrt{s}=$ $17 \mathrm{GeV}$ and $40 \mathrm{mb}$ at $\sqrt{s}=130 \mathrm{GeV}$.

With the same parametrization of the initial trans- 
verse density profile we compute the initial transverse source radii parallel $\left(R_{x}(b)=\left\langle x^{2}\right\rangle^{1 / 2}\right)$ and perpendicular $\left(R_{y}(b)=\left\langle y^{2}\right\rangle^{1 / 2}\right)$ to the impact parameter. These as well as the initial spatial deformation

$$
\epsilon_{x}(b) \equiv \delta(b)=\frac{R_{y}^{2}-R_{x}^{2}}{R_{y}^{2}+R_{x}^{2}}
$$

are shown in Fig. 1 for $\mathrm{Au}+\mathrm{Au}$ at $\sqrt{s}=130 \mathrm{AGeV}$ as functions of the scaled impact parameter $b / R$.

Particles are initially produced azimuthally symmetric in momentum space. The initial deformation in coordinate space generates an azimuthally asymmetric momentum distribution if and only if the produced particles rescatter off each other. The more rescatterings take place and the larger the initial deformation $\delta$, the more anisotropic the final momentum distribution will be. A quantitative measure of this anisotropy is provided by its harmonic coefficients $v_{n}$ in an event-by-event Fourier expansion with respect to the azimuthal angle $\phi$ 24. $v_{2}$ is called the "elliptic flow coefficient".

To relate the initial spatial anisotropy $\delta$ to the measured momentum anisotropy $v_{2}$ one has to model the interactions during the fireball expansion. The initial average density and source size is larger in central than in peripheral collisions. The particle mean free paths $\lambda_{\mathrm{mfp}}=1 /(\sigma \rho)$, where $\sigma$ is the scattering cross section and $\rho$ the time-dependent average density of scatterers, vary from a few fm or less initially to infinity at freeze-out. Since the ratio $\lambda_{\mathrm{mfp}} / R_{x, y}$ is smaller over a longer time in central than in peripheral collisions, hydrodynamic models are more likely to work in central or semicentral collisions, especially at high energies where the initial particle densities are large. The low density limit (LDL), on the other hand, should apply to peripheral collisions, especially at low energies with small initial particle densities.

3. The Hydrodynamic Limit.- The full hydrodynamic treatment of a non-central collision is a tedious $3+1$ dimensional problem 25,10,26. We reduce the complexity of the task to $2+1$ dimensions by assuming boostinvariant longitudinal flow [6 9]. This assumption limits our description to a region around midrapidity which is, however, expected to grow as the collision energy increases.

The evolution of a hydrodynamical system is determined by its initial conditions and equation of state (EOS). We fix the initial conditions as in [7, 8$]$ by requiring a good fit to the $p_{t}$-spectra of protons and negatively charged particles in central $\mathrm{Pb}+\mathrm{Pb}$ collisions at the SPS [7]. The SPS initial conditions are scaled to the RHIC energy of $\sqrt{s}=130 \mathrm{AGeV}$ by adjusting the initial energy density $\epsilon_{0}$ (keeping the product $T_{0} \tau_{0}$ of the initial temperature and thermalization time fixed \&) until the final charged particle pseudorapidity density at midrapidity agrees with the published measurement by the PHOBOS Collaboration [27. The initial baryon density was chosen to give the ratio $\bar{p} / p=0.65$ in the final state 28]. Adjusting the initial baryon density at fixed initial energy density has no measurable consequences for the developing flow pattern, since the pressure is insensitive to $n_{b}$ when the latter is small [8].

\begin{tabular}{|c|c|c|c|c|c|c|}
\hline & \multicolumn{3}{|c|}{ SPS } & \multicolumn{3}{|c|}{ RHIC } \\
\hline$T_{\mathrm{f}}(\mathrm{MeV}) \approx$ & 120 & 120 & 140 & 120 & 140 & 140 \\
\hline$\epsilon_{\mathrm{f}}\left(\mathrm{GeV} / \mathrm{fm}^{3}\right)$ & 0.06 & 0.06 & 0.15 & 0.05 & 0.14 & 0.14 \\
\hline $\mathrm{EOS}$ & $\mathrm{Q}$ & $\overline{\mathrm{H}}$ & $\overline{\mathrm{H}}$ & $\mathrm{Q}$ & $\mathrm{Q}$ & $\overline{\mathrm{H}}$ \\
\hline$\epsilon_{0}\left(\mathrm{GeV} / \mathrm{fm}^{3}\right)$ & 9.0 & 9.0 & 10.0 & 23.0 & 23.0 & 22.3 \\
\hline$n_{b, 0}\left(\mathrm{fm}^{-3}\right)$ & 1.1 & 1.1 & 1.2 & 0.12 & 0.25 & 0.25 \\
\hline$\tau_{0}(\mathrm{fm} / \mathrm{c})$ & 0.8 & 0.8 & 0.8 & 0.6 & 0.6 & 0.6 \\
\hline$T_{0}(\mathrm{MeV})$ & 257 & 238 & 242 & 334 & 334 & 270 \\
\hline$d N_{\mathrm{ch}} / d y(b=0)$ & 390 & 370 & 420 & 670 & 690 & 685 \\
\hline$d N_{\mathrm{ch}} /\left.d y\right|_{y=0}$ & 355 & 335 & 385 & 615 & 630 & 625 \\
\hline$d N_{\mathrm{ch}} /\left.d \eta\right|_{|\eta|<1}$ & 310 & 290 & 325 & 545 & 545 & 545 \\
\hline
\end{tabular}

Table 1. Freeze-out temperatures, equations of state and initial conditions for central $(b=0)$ collisions employed for the hydrodynamical calculations shown in this paper. The last two rows show the final charged particle multiplicity densities in rapidity and pseudorapidity for the $6 \%$ most central collisions, to facilitate comparison with the PHOBOS data [27.

We use two different equations of state to check how the quark-hadron phase transition or its absence affects the flow anisotropy. EOS Q has a first order phase transition to QGP at $T_{\mathrm{c}}=165 \mathrm{MeV}$ whereas EOS H contains only hadronic resonances at all densities. At RHIC energies EOS $\mathrm{H}$ leads to unrealistic particle densities in the initial state. However, this EOS gives an impression how a system without a phase transition would behave; this is important when trying to separate specific phase transition signatures [7, 8, from generic hydrodynamical features. Further details of the construction of these equations of state can be found in [29].

There is still no final consensus at which temperature the hydrodynamical description breaks down at SPS energies [30], nor is there any a priori reason why this temperature should be the same at SPS and RHIC. In our earlier studies of $\mathrm{Pb}+\mathrm{Pb}$ collisions at the SPS [31] we found $\mathrm{EOS} \mathrm{Q}$ to favour a lower freeze-out temperature $\left(T_{\mathrm{f}} \approx 120 \mathrm{MeV}\right)$ than EOS $\mathrm{H}\left(T_{\mathrm{f}} \approx 140 \mathrm{MeV}\right)$. Still, a fit of the pion and proton $p_{t}$ spectra from $158 \mathrm{AGeV}$ $\mathrm{Pb}+\mathrm{Pb}$ collisions 32 with $\operatorname{EOS~} \mathrm{H}$ and $T_{\mathrm{f}} \approx 120 \mathrm{MeV}$ looks quite acceptable 33. Therefore we do the SPS calculations using EOS Q with freeze-out energy density $\epsilon_{\mathrm{f}}=0.06 \mathrm{GeV} / \mathrm{fm}^{3}$ (corresponding to $T_{\mathrm{f}} \approx 120 \mathrm{MeV}$ ), and we probe the effect of the freeze-out temperature on elliptic flow by using EOS $\mathrm{H}$ with freeze-out energy densities $\epsilon_{\mathrm{f}}=0.06$ as well as $0.15 \mathrm{GeV} / \mathrm{fm}^{3}$ (the latter corresponds to $\left.T_{\mathrm{f}} \approx 140 \mathrm{MeV}\right)$.

At RHIC the baryon number density at freeze-out is much smaller than at the SPS, and the energy densities corresponding to $T_{\mathrm{f}}=120$ and $140 \mathrm{MeV}$ are $\epsilon_{\mathrm{f}} \approx 0.05$ and $0.14 \mathrm{GeV} / \mathrm{fm}^{3}$, respectively. We do the RHIC calculations for EOS $\mathrm{Q}$ with both of these freeze-out en- 
ergy densities and for EOS $\mathrm{H}$ with freeze-out density $\epsilon_{\mathrm{f}}=0.14 \mathrm{GeV} / \mathrm{fm}^{3}$. To convert the fluid variables to particle and resonance distributions we employ the CooperFrye freeze-out prescription [34]. Subsequent resonance decays are calculated using the decay kinematics described in [35]. The elliptic flow parameters $v_{2}\left(p_{t}\right)$ and $v_{2}$ are obtained by Fourier expanding the calculated differential and $p_{t}$-integrated momentum distributions. For $T_{\mathrm{f}} \approx 140 \mathrm{MeV}$ decay contributions reduce the elliptic flow of pions by $18-25 \%$ depending on the EOS and impact parameter; the corresponding decrease at $T_{\mathrm{f}} \approx 120 \mathrm{MeV}$ is $8-15 \%$.

The $p_{t}$-averaged elliptic flow $v_{2}$ as a function of collision centrality is shown in Fig. 2 for $\mathrm{Pb}+\mathrm{Pb}$ at the SPS and in Fig. 3 for $\mathrm{Au}+\mathrm{Au}$ at RHIC. At the SPS energy the calculation is for midrapidity pions of all $p_{t}$, [4] whereas the RHIC results include all charged particles with $|\eta|<1.3$ and $0.1<p_{t}<2 \mathrm{GeV} / c$ [5]. We have used the same centrality measures (impact parameter $b$ at the SPS, the fractional charged particle multiplicity density at midrapidity $N_{\mathrm{ch}} / N_{\max }$ at RHIC) as in the corresponding experimental publications. []

At the SPS energy the hydrodynamical calculation can reproduce the data only for the most central collisions. Already in semi-central collisions the calculations overpredict the measured elliptic flow significantly, and the disagreement increases to about a factor 2 in peripheral collisions. However, the comparison in Fig. 2 should be viewed with some care: as stated above, our calculation applies only to midrapidity $\left(y_{1 \mathrm{ab}}=2.9\right)$ whereas the statistically more significant open squares correspond to an average over the forward hemisphere. The preliminary data on the rapidity dependence of $v_{2}$ reported in [4] show non-trivial structures which are averaged out in the open squares. On the other hand, when taking into account the $p_{t}$ cut in [3], one finds that the midrapidity

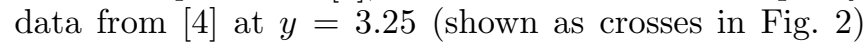
are significantly lower than the extrapolation of $v_{2}$ to midrapidity published in [3]. A reliable measurement of $v_{2}$ at midrapidity is thus presently not available for this

\footnotetext{
${ }^{1}$ In 10 resonance decays were found to reduce the pion elliptic flow by $40 \%$. However, in that work only pions with $50<p_{t}<350 \mathrm{MeV} / \mathrm{c}$ were taken into account whereas we include either all $p_{t}$ (SPS) or only cut out $p_{t}<100 \mathrm{MeV} / c$ (RHIC). Resonance decays reduce the pion elliptic flow especially at low values of $p_{t}$; using similar $p_{t}$ cuts as in 10 at SPS energies our results are compatible.

${ }^{2}$ To compare with the RHIC data, we had to scale $N_{\max }$ by a factor 0.95 since the hydrodynamic calculation cannot account for fluctuations in the charged multiplicity at fixed impact parameter: while our "wounded nucleon" initialization nicely describes the measured multiplicity distribution [5] up to the "knee" at $b=0$, the calculated distribution drops there abruptly to zero while the data show fluctuations up to a value $N_{\max }$ which is $\approx 5 \%$ larger.
}

collision system, but would be very welcome.

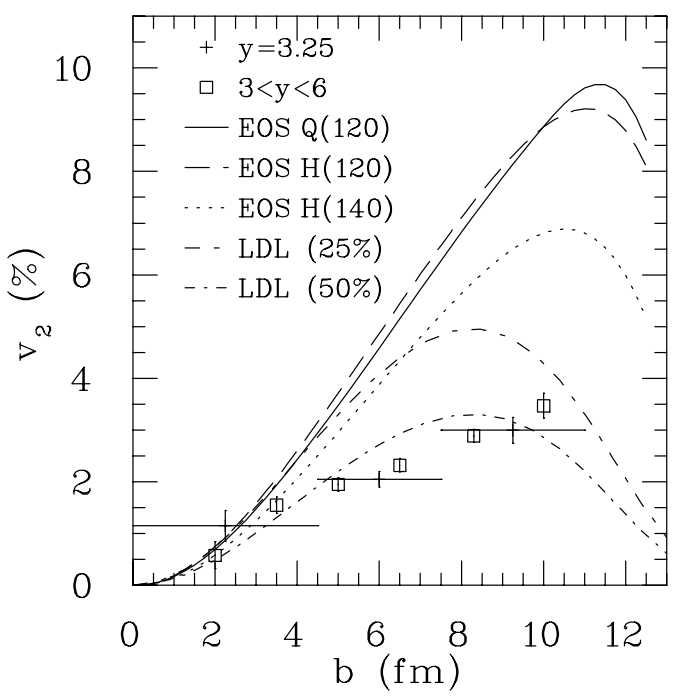

FIG. 2. Elliptic flow for pions at midrapidity vs. centrality, for $158 A \mathrm{GeV} \mathrm{Pb}+\mathrm{Pb}$ collisions. Hydrodynamic calculations and results from the LDL are compared to NA49 data [3, 4. 4 . Numbers in brackets give $T_{\mathrm{f}}$ in $\mathrm{MeV}$ and the reduction from resonance decays, respectively.

Taking the available data at face value, we are unable to account for them within the hydrodynamic approach even when varying the initial conditions, the EOS and the freeze-out temperature within the constraints provided by the measured single particle distributions. Several examples are shown in Fig. 2 .

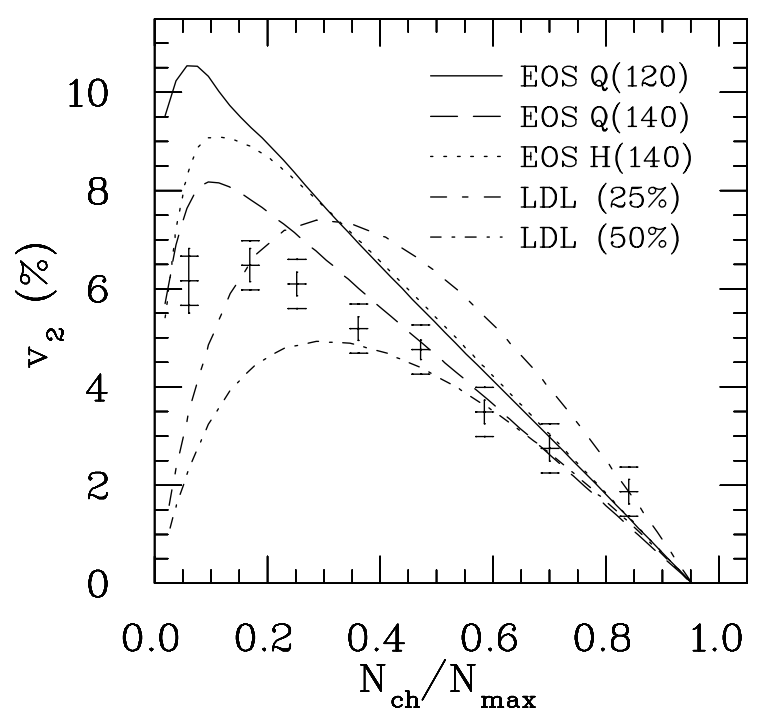

FIG. 3. Centrality dependence of the elliptic flow coefficient $v_{2}$ for charged particles from $\mathrm{Au}+\mathrm{Au}$ collisions at $\sqrt{s}=130 \mathrm{AGeV}$. The data [0] are shown with the quoted systematic error of \pm 0.005 . For details see text.

Fig. 3 shows that, on the other hand, hydrodynamics successfully reproduces the elliptic flow measured at 
RHIC for central and semi-central collisions. The best agreement is reached for EOS $Q$ with $T_{\mathrm{f}}=140 \mathrm{MeV}$, where discrepancies begin to be significant only at impact parameters above $7 \mathrm{fm}$, but stay below $20 \%$ even for the most peripheral collisions. Lower freeze-out temperatures or the use of EOS $\mathrm{H}$ (which is effectively harder at these collision energies, where the expanding matter spends a large fraction of its total lifetime in the soft phase transition region [8]) predict somewhat larger elliptic flow. They also give larger radial flow; when singleparticle spectra from this experiment become available, they will provide a crucial test of the approach and remove the ambiguity of the freeze-out temperature 36.

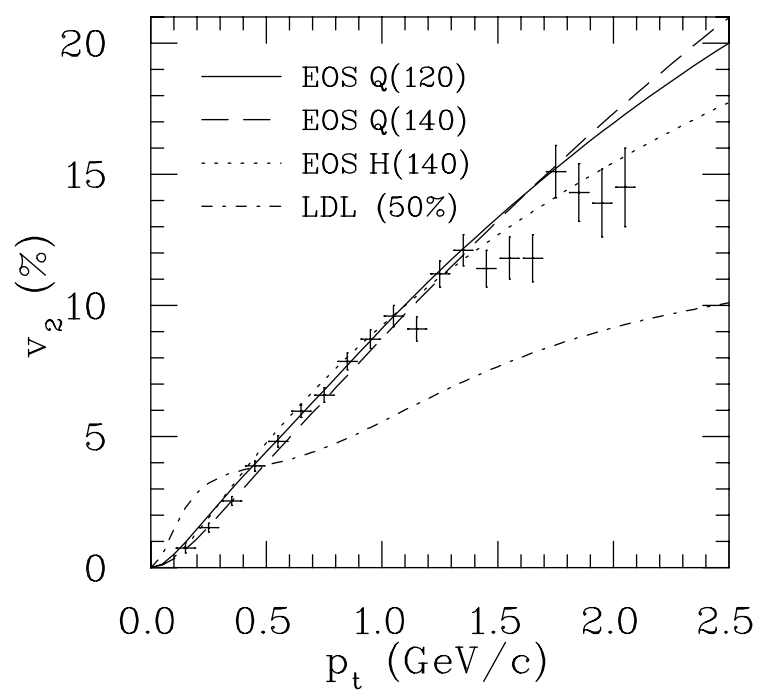

FIG. 4. The elliptic flow of charged particles from $\mathrm{Au}+\mathrm{Au}$ collisions at $\sqrt{s}=130 A \mathrm{GeV}$ vs. transverse momentum. Hydrodynamic calculations and predictions from the LDL are compared with the data [5]. The shape of the LDL curve reflects the weighting of different hadrons with their contribution to the charged particle spectrum; at small $p_{t}$ it is dominated by light pions, at high $p_{t}$ by heavy baryons.

Fig. 1 shows the $p_{t}$-dependence of elliptic flow for minimum bias $\mathrm{Au}+\mathrm{Au}$ collisions at RHIC [5]. Following the prescription in [5] it is calculated by

$$
v_{2}\left(p_{t}\right)=\frac{\int b d b v_{2}\left(p_{t} ; b\right) \frac{d N_{\mathrm{ch}}}{d y p_{t} d p_{t}}(b)}{\int b d b \frac{d N_{\mathrm{ch}}}{d y p_{t} d p_{t}}(b)}
$$

with a cut-off at $b_{\max }=13.5 \mathrm{fm}$. The agreement between the data and the hydrodynamical calculations is excellent, especially when one considers the small variations of the latter upon changing parameters within the range allowed by the constraints. Only for $p_{t}$ above about 1.5 $\mathrm{GeV} / c$ does the measured elliptic flow lag behind the hydrodynamic prediction, indicating a departure from thermalization for high- $p_{t}$ particles. In future it will be interesting to follow the data to higher $p_{t}$ where $v_{2}$ is first expected to saturate due to lack of thermalization, before decreasing again as expected from jet quenching
[21]. The hydrodynamic curves start out quadratically at low $p_{t}$, as required by general principles [37, then quickly 36] turn over to an approximately linear rise and keep increasing monotonically with $p_{t}$, eventually saturating at $v_{2}\left(p_{t}\right)=1$ as $p_{t} \rightarrow \infty$.

It is not immediately obvious how the good agreement between theory and data in Fig. 1 is compatible with the clearly visible discrepancies in Fig. 3 for (semi-)peripheral collisions. One possibility is that the impact parameter dependence 22] of the charged particle multiplicity (i.e. of the normalization of the spectra which enter the weighting procedure (2)) is different in theory and experiment. This can be settled by measuring the dependence of $d N_{\mathrm{ch}} / d y$ on the number of participants $N_{\text {part }}$ 22, 23, 38, and by providing $v_{2}\left(p_{t}\right)$ for different centrality bins. A more likely explanation is suggested by the observation that the clearly visible differences in Fig. 3 resulting from varying the EOS and $T_{\mathrm{f}}$ are much smaller in Fig. 4. An analysis shows that the variations in Fig. 3 result from different slopes of the single particle spectra which, when averaging the nearly identical hydrodynamic curves in Fig. A over $p_{t}$, give different relative weights to the regions of small and large $v_{2}$. The lower $p_{t}$-averaged $v_{2}$ for peripheral collisions could thus be due to steeper single-particle spectra in peripheral collisions than predicted by the model, e.g. due to earlier freeze-out at higher $T_{\mathrm{f}}$ and smaller radial flow 20. This can be clarified by measuring the single-particle spectra [36] and the $p_{t}$-dependence of elliptic flow at different centralities.

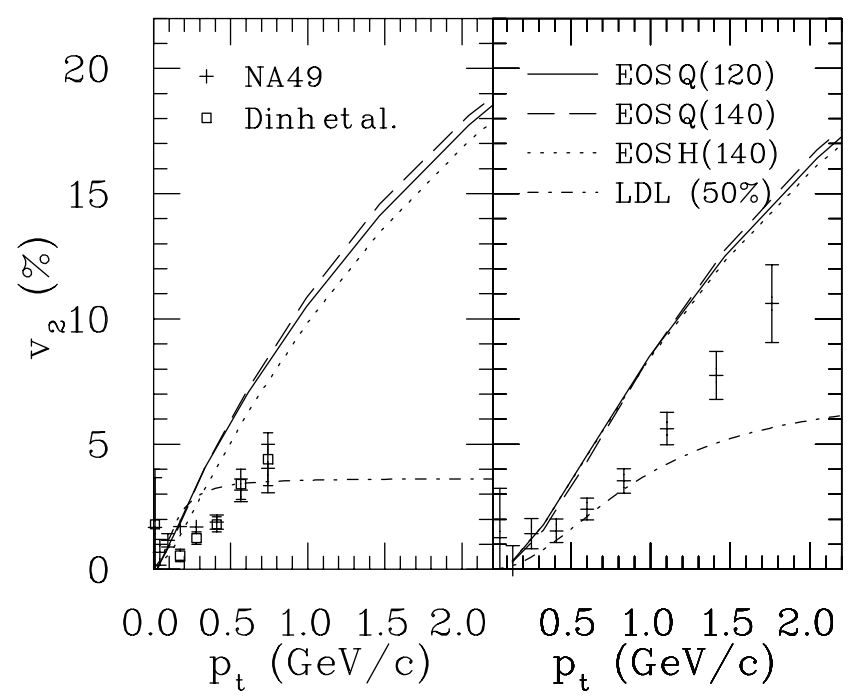

FIG. 5. $p_{t}$-dependence of elliptic flow for pions (left) and protons (right) from $\mathrm{Pb}+\mathrm{Pb}$ collisions at $\sqrt{s}=17 \mathrm{~A} \mathrm{GeV}$ with impact parameters $b<11 \mathrm{fm}$. The data 3] correspond to $6.5 \mathrm{fm}<b<8 \mathrm{fm}$ and are averaged over the forward rapidity interval $4<y<5$ while the hydrodynamic calculations apply to midrapidity $y=2.9$. The squares show the NA49 data after correction for azimuthal HBT correlations [39]. For details see text.

The $p_{t}$-dependence of $v_{2}$ for pions and protons from 
semi-central $\mathrm{Pb}+\mathrm{Pb}$ collisions at the SPS is shown in Fig. 5. Unfortunately, no midrapidity data are available, and a meaningful comparison between theory and experiment of the magnitude of $v_{2}$ is thus not possible. We note, however, that the data show the same approximately linear rise of $v_{2}$ with $p_{t}$ and the smaller elliptic flow at small $p_{t}$ for protons than for pions as predicted by hydrodynamics. Such a linear rise is inconsistent with the Low Density Limit (LDL) (see below); it remains to be seen to what extent realistic kinetic codes are able to reproduce a linear rise of $v_{2}\left(p_{t}\right)$, albeit with a smaller slope than predicted by hydrodynamics, at SPS energies.

4. The Low Density Limit. - If the nuclear overlap zone is small and the initial density not very large, produced particles can escape from the reaction zone suffering only a few reinteractions. In the extreme limit the system is streaming freely, and no collective flow builds up at all. For sufficiently dilute systems the elliptic flow can be calculated from the first order correction to free streaming arising from particle collisions. Such a perturbative approach is valid as long as the particle mean free paths $\lambda_{\text {mfp }}$ are larger than the overlap zone $R_{x, y}(b)$.

In the low density limit (LDL) the effect of scatterings was calculated to first order by inserting the free streaming distribution into the Boltzmann collision term [11]. The resulting momentum space anisotropy leads to an elliptic flow coefficient $v_{2}^{i}$ for particle species $i$, scattering with particles $j(i, j=\pi, p, K, \ldots)$, given by [11]

$$
v_{2}^{i}\left(b, p_{t}\right)=\frac{v_{i \perp}^{2}\left(p_{t}\right)}{16 \pi} \frac{\delta(b)}{R_{x}(b) R_{y}(b)} \sum_{j} \frac{\left\langle v_{i j} \sigma_{\mathrm{tr}}^{i j}\right\rangle}{\left\langle v_{i j \perp}^{2}\right\rangle} \frac{d N_{j}}{d y}(b) \text {. }
$$

Here, $v_{i \perp}\left(p_{t}\right)$ is the transverse velocity of a particle $i$ with momentum $p_{t}$, and $v_{i j}$ is the relative velocity between particle $i$ and scatterer $j$. Brackets $\langle\ldots\rangle$ denote averaging over scatterer momenta $p_{j} . \quad \sigma_{\mathrm{tr}}^{i j}$ is the transport cross section responsible for momentum transfer. Similar to the hydrodynamic limit, the elliptic flow coefficient is proportional to the initial spatial deformation. Its $p_{t}$-dependence is, however, quite different, saturating at large $p_{t}$ (when $v_{i \perp} \rightarrow c$ ) at values much below 1 . At small $p_{t}, v_{2}$ rises quadratically as it should [37], at a rate which is directly given by the asymptotic saturation level. This coupling between the curvature at small $p_{t}$ and the saturation level at large $p_{t}$ does not permit a sustained approximately linear rise of $v_{2}$ in the intermediate $p_{t^{-}}$ region.

Let us now try to quantify the parameters entering the LDL formula (3) for SPS and RHIC energies. $\delta(b)$ and $R_{x, y}(b)$ are obtained from Fig. 11. We assume that in the region of applicability of the LDL the matter can be described with hadronic degrees of freedom. For isotropic scattering the transport cross sections are about half of the total cross section. However, the produced pions are fast and scatter off other pions mostly in the forward direction; the same is true for p-wave $\rho$ - and $\Delta$-resonance scattering. We estimate that the angleaveraged cross sections are only $\simeq\left\langle\sin ^{2} \theta \cos \theta\right\rangle=1 / 4$ of the total ones, averaged over the relevant relative momentum range. For these latter we assume $\sigma^{\pi \mathrm{M}} \simeq 10 \mathrm{mb}$, $\sigma^{\pi \mathrm{B}}=\sigma^{p \mathrm{M}} \simeq 30 \mathrm{mb}$, and $\sigma^{p \mathrm{~B}} \simeq 40 \mathrm{mb}$ where $\mathrm{M}$ and $\mathrm{B}$ stand for arbitrary mesonic and baryonic resonances.

If the cross sections were the same for all particles we could replace the sum over $d N_{j} / d y$ by $\frac{3}{2} \times d N_{\mathrm{ch}} / d y$. We use $d N_{\mathrm{ch}} / d y \simeq 410$ in central $\mathrm{Pb}+\mathrm{Pb}$ collisions at the $\operatorname{SPS}(\sqrt{s}=17 A \mathrm{GeV})$ and $d N_{\mathrm{ch}} / d y \simeq 630$ for central $\mathrm{Au}+\mathrm{Au}$ collisions at $\mathrm{RHIC}(\sqrt{s}=130 \mathrm{AGeV})$ 27]. $[d N / d y$ at midrapidity is about $15 \%$ larger than $d N /\left.d \eta\right|_{|\eta|<1}$.] Since the baryon and anti-baryon cross sections are larger, their rapidity densities are required separately; we use $d N_{B+\bar{B}}^{\mathrm{SPS}} / d y \simeq 110$ for central $\mathrm{Pb}+\mathrm{Pb}$ collisions at the SPS 400 and estimate roughly $d N_{B+\bar{B}}^{\mathrm{RHIC}} / d y \simeq$ 70 for central $\mathrm{Au}+\mathrm{Au}$ collisions at RHIC. For the centrality dependence we assume that the rapidity densities scale with the number of participants as shown in Fig. 1.

All relative velocities are assumed to be of the order of the speed of light. To obtain the $p_{t}$-averaged elliptic flow we average over $v_{i \perp}$ with an exponential $m_{t}$-distribution with inverse slope of $130 \mathrm{MeV}$; this gives $\left\langle v_{\perp}^{2}\right\rangle=0.68$ for pions and $\left\langle v_{\perp}^{2}\right\rangle=0.22$ for protons. In the RHIC data only charged particles with $p_{t}>100 \mathrm{MeV} / c$ are included, which we try to take into account by increasing $\left\langle v_{\perp}^{2}\right\rangle$ to 0.75 (assuming pion dominance).

Finally, we have to correct for resonance decay contributions. The actual rapidity density of scatterers in the reaction zone is smaller than the observed $d N / d y$ since a large fraction of the latter comes from unstable resonances which only decay after the $v_{2}$-generating rescatterings have happened. We estimate the corresponding reduction factor for the density of scatterers (and thus for $v_{2}$ ) to be about a factor 2. For illustration of the systematic uncertainties we also show curves where only $25 \%$ of the final charged multiplicity arise from resonance decays. Clearly, all these numbers are very rough, and our estimates for the factor multiplying $v_{i \perp}^{2}$ in (3) could be easily off by $50 \%$ in both directions.

Curves showing the elliptic flow from the LDL are included in Figs. 2 5. The LDL gives about 50\% more elliptic flow at RHIC than at the SPS. This reflects mostly the corresponding ratio of the charged multiplicity densities as shown by the following expression (see Eq. (3)):

$$
\frac{v_{2}^{\mathrm{RHIC}}}{v_{2}^{\mathrm{SPS}}}=\frac{d N_{\mathrm{ch}}^{\mathrm{RHIC}} / d y+c_{\mathrm{RHIC}} d N_{B+\bar{B}}^{\mathrm{RHIC}} / d y}{d N_{\mathrm{ch}}^{\mathrm{SPS}} / d y+c_{\mathrm{SPS}} d N_{B+\bar{B}}^{\mathrm{SPS}} / d y} .
$$

The constant is given by $c=(2 / 3)\left(\sigma_{\mathrm{tr}}^{\pi \mathrm{B}} / \sigma_{\mathrm{tr}}^{\pi \mathrm{M}}-1\right) \approx 4 / 3$. While the LDL thus happens to be able to reproduce the impact parameter dependence of the $p_{t}$-averaged elliptic flow at the SPS (Fig. 2), it slightly underpredicts the same quantity at RHIC (Fig. 3). Inspection of the $p_{t}$-dependence of $v_{2}$ in minimum bias events (which is dominated by semi-central collisions) reveals, however, that the LDL gets it completely wrong at RHIC energies (Fig. 4). Fig. 5 shows that, while the pion flow data at the SPS are inconclusive due to their limited $p_{t}$-coverage, the 
$p_{t}$-dependence of $v_{2}$ for protons from semi-central $\mathrm{Pb}+\mathrm{Pb}$ collisions at the SPS is again incompatible with the LDL. Whereas the hydrodynamic model has difficulties reproducing the slope (which may, however, be due to the different rapidity windows in the data and the model), the LDL gives a completely wrong shape.

That the RHIC data for semicentral collisions far exceed the LDL prediction demonstrates that first collisions are insufficient and multiple collisions are required. The agreement with hydrodynamics except for a small deficiency for $p_{t} \gtrsim 1.5 \mathrm{GeV} / c$ indicates that the system is very close to local thermal equilibrium. For very peripheral collisions we expect that departures from the hydrodynamical prediction set in at lower $p_{t}$-values; it will be interesting to see whether experiment confirms this.

5. Conclusions. - The recent elliptic flow data from $\mathrm{Au}+\mathrm{Au}$ collisions at $\mathrm{RHIC}$ show remarkable quantitative agreement with the hydrodynamical model, indicating a large degree of thermalization in the earliest collision stages, well before hadronization. With initial conditions tuned to data from central collisions at the SPS and no additional adjustment of parameters except for a simple scaling of the charged multiplicity to the value measured by PHOBOS, the hydrodynamic model reproduces quantitatively the centrality dependence of $v_{2}$ up to impact parameters of about $7 \mathrm{fm}$ and its $p_{t}$-dependence up to transverse momenta of about $1.5 \mathrm{GeV} / c$. Deviations occur only in very peripheral collisions and for particles with $p_{t}>1.5 \mathrm{GeV} / c$; they may be due to a combination of incomplete early thermalization [5] and/or earlier freeze-out [20] in these kinematic regions. The low density limit LDL roughly reproduces the shape of the centrality dependence of $v_{2}$ at RHIC, but slightly underpredicts the magnitude of the $p_{t}$-averaged elliptic flow and fails badly for the shape of its $p_{t}$-dependence. It works better for the centrality dependence of $v_{2}$ at the SPS, but again cannot describe the observed nearly linear $p_{t^{-}}$ dependence of proton elliptic flow. The hydrodynamic model gets the shape of all the $v_{2}$ distributions at the SPS right, but seems to overpredict the absolute magnitude of $v_{2}$; this last statement is, however, uncertain due to the lack of reliable midrapidity data from $\mathrm{Pb}+\mathrm{Pb}$ collisions at the SPS.

These findings suggest that at RHIC thermalization sets in very early (the hydrodynamic simulations point to a thermalization time scale of less than $1 \mathrm{fm} / c$ ), but that it may take longer at the SPS. A better understanding of the onset of deviations from hydrodynamic behaviour at RHIC, which should be provided by future measurements of $d N_{\mathrm{ch}} / d y, v_{2}\left(p_{t}\right)$, and the single-particle $p_{t}$-spectra as functions of the number of participants, will yield crucial insights into the kinetic evolution at the earliest collision stages. Existing parton [12,13 and hadron [19] cascade calculations reproduce the approximate linear rise of $v_{2}\left(p_{t}\right)$ up to $p_{t} \lesssim 500 \mathrm{MeV} / c$, but at higher $p_{t}$ the elliptic flow levels off, and in UrQMD its absolute value at RHIC, averaged over $p_{t}$, is underpredicted by about a factor 4-5 19]. The parton cascade MPC [13] builds up elliptic flow earlier, but quantitatively does not perform very much better. This raises serious questions about the adequacy of incoherent scattering among on-shell particles to describe the early collision stage and the approach to thermalization in ultrarelativistic heavy-ion collisions.

The accurate agreement of the STAR data [5] with hydrodynamic predictions [8] proves that with elliptic flow one has found a hadronic signature which is sensitive to the hot and dense quark-gluon plasma stage before hadronization sets in. More detailed measurements like those mentioned above (in particular the shape of the single-particle spectra) should help to confirm and further constrain the picture [36]. This will open the door to quantitatively characterize the QGP equation of state and in particular to distinguish between equations of state with and without a phase transition [8].

We gratefully acknowledge many fruitful discussions with M. Bleicher, V. Koch, A. Poskanzer, P.V. Ruuskanen, R. Snellings, S. Voloshin, and N. Xu. P.K. wishes to thank both LBNL and BNL for hospitality while finishing this paper. This work was supported in part by the Director, Office of Science, Office of High Energy and Nuclear Physics, Division of Nuclear Physics, and by the Office of Basic Energy Sciences, Division of Nuclear Sciences, of the U.S. Department of Energy under Contract No. DE-AC03-76SF00098.

[1] W. Reisdorf and H.G. Ritter, Ann. Rev. Nucl. Part. Sci. 47 (1997) 663.

[2] J. Barrette et al. (E877 Collaboration), Phys. Rev. C56 (1997) 3254; H. Liu et al. (E895 Collaboration), Nucl. Phys. A638 (1998) 451c.

[3] H. Appelshäuser et al. (NA49 Collaboration), Phys. Rev. Lett. 80 (1998) 4136 (in the present work the updated figures archived on the NA49 home page http://na49info.cern.ch/na49/Archives/Images/Publications 1 were used)

[4] A. Poskanzer, S. Voloshin et al. (NA49 Collaboration), Nucl. Phys. A661 (1999) 341c.

[5] K. H. Ackermann et al. (STAR Collaboration), nuclex/0009011

[6] J.Y. Ollitrault, Phys. Rev. D 46 (1992) 229; ibid. 48 (1993) 1132; and Nucl. Phys. A638 (1998) 195c.

[7] P.F. Kolb, J. Sollfrank, and U. Heinz, Phys. Lett. B 459 (1999) 667; P.F. Kolb, J. Sollfrank, P.V. Ruuskanen and U. Heinz, Nucl. Phys. A661 (1999) 349c.

[8] P.F. Kolb, J. Sollfrank, and U. Heinz, Phys. Rev. C 62 (2000) 054909.

[9] D. Teaney and E.V. Shuryak, Phys. Rev. Lett. 83 (1999) 4951.

[10] T. Hirano, nucl-th/9904082, nucl-th/0004029; T. Hirano, K. Tsuda, and K. Kajimoto, nucl-th/0011087.

[11] H. Heiselberg and A. Levy, Phys. Rev. C 59 (1999) 2716.

[12] B. Zhang, M. Gyulassy, and C.M. Ko, Phys. Lett. B 455 
(1999) 45 .

[13] D. Molnar, talk presented at the 30th International Workshop on Multiparticle Dynamics (ISMD2000), Tihany, Hungary, 9.-15. Oct. 2000, to appear in the proceedings.

[14] H. Sorge, Phys. Rev. Lett. 78 (1997) 2309.

[15] R.J.M. Snellings, A.M. Poskanzer and S.A. Voloshin, STAR note 388, nucl-ex/9904003.

[16] H. Liu, S. Panitkin, and N. Xu, Phys. Rev. C59 (1999) 348.

[17] L.V. Bravina, A. Faessler, C. Fuchs, and E.E. Zabrodin, Phys. Rev. C 61 (2000) 064902.

[18] S. Soff, S.A. Bass, M. Bleicher, H. Stöcker and W. Greiner, nucl-th/9903061.

[19] M. Bleicher and H. Stöcker, hep-ph/0006147.

[20] D. Teaney. J. Lauret, and E. Shuryak, nucl-th/0011058.

[21] X.N. Wang, nucl-th/0009019.

[22] P. Huovinen, P. Kolb, U. Heinz, and K.J. Eskola, in preparation.

[23] K.J. Eskola, K. Kajantie, and K. Tuominen, hep$\mathrm{ph} / 0009246$.

[24] S.A. Voloshin and Y. Zhang, Z. Phys. C 70 (1996) 665.

[25] D.H. Rischke, S. Bernard, and J.A. Maruhn, Nucl. Phys. A595 (1995) 346; D.H. Rischke, Y. Pürsün, and J.A. Maruhn, ibid. A595 (1995) 383.

[26] C. Nonaka, N. Sasaki, S. Muroya, and O. Miyamura, Nucl. Phys. A661 (1999) 353c; K. Morita, S. Muroya, H. Nakamura, and C. Nonaka, Phys. Rev. C 61 (2000) 034904; C. Nonaka, E. Honda, and S. Muroya, hep$\mathrm{ph} / 0007187$.

[27] B.B. Back et al. (PHOBOS collaboration), Phys. Rev. Lett. 85 (2000) 3100.

[28] Preliminary result reported by the STAR Collaboration at DNP2000, see http://www-rnc.lbl.gov/STAR/conf/ talks2000/dnp/ullrich.html

[29] J. Sollfrank et al., Phys. Rev. C 55 (1997) 392.

[30] B. Kämpfer et al., J. Phys. G 23 (1997) 2001; H. Appelshäuser et al. (NA49 Collaboration), Eur. Phys. J. C 2 (1998) 661; B. Tomášik, U.A. Wiedemann, and U. Heinz, nucl-th/9907096; T. Peitzmann, nucl-th/0006025.

[31] P. Huovinen, P.V. Ruuskanen, and J. Sollfrank, Nucl. Phys. A650 (1999) 227.

[32] H. Appelshäuser et al. (NA49 Collaboration), Phys. Rev. Lett. 82 (1999) 2471.

[33] P.F. Kolb, diploma thesis, University of Regensburg, 1999, unpublished.

[34] F. Cooper and G. Frye, Phys. Rev. D 10 (1974) 186.

[35] J. Sollfrank, P. Koch, and U. Heinz, Z. Phys. C 52 (1991) 593.

[36] P. Huovinen, P. Kolb, U. Heinz, S.A. Voloshin, and P.V. Ruuskanen, in preparation.

[37] P. Danielewicz, Phys. Rev. C 51 (1995) 716.

[38] X.N. Wang and M. Gyulassy, nucl-th/0008014.

[39] M. Dinh, N. Borghini, and J.-Y. Ollitrault, Phys. Lett. B 477 (2000) 51.

[40] P.G. Jones et al. (NA49 Collaboration), Nucl. Phys. A610 (1996) 188c; G. Roland et al. (NA49 Collaboration), Nucl. Phys. A638 (1998) 91c; F. Sikler et al. (NA49 Collaboration), Nucl. Phys. A661 (1999) 45c. 